\title{
High expression of AFAP1-AS1 is associated with poor survival and short-term recurrence in pancreatic ductal adenocarcinoma
}

Yibiao Ye ${ }^{1 \dagger}$, Jie Chen ${ }^{1 \dagger}$, Yu Zhou ${ }^{2,5}$, Zhiqiang Fu², Quanbo Zhou ${ }^{2}$, YingXue Wang ${ }^{2}$, Wenchao Gao ${ }^{2}$, ShangYou Zheng ${ }^{2}$, Xiaohui Zhao ${ }^{3}$, Tao Chen ${ }^{1 *}$ and Rufu Chen ${ }^{2,4^{*}}$

\begin{abstract}
Background: Pancreatic ductal adenocarcinoma (PDAC) is still a lethal malignancy. Long noncoding RNAs (IncRNAs) have been shown to play a critical role in cancer development and progression. Here we identified overexpression of the IncRNA AFAP1-AS1 in PDAC patients and evaluated its prognostic and functional relevance.

Methods: The global IncRNA expression profile in PDAC was measured by IncRNA microarray. Expression of AFAP1-AS1 was evaluated by reverse-transcriptase quantitative polymerase chain reaction (RT-qPCR) in 90 PDAC tissue samples and adjacent normal tissues. The impact of AFAP1-AS1 expression on cell proliferation, migration, and invasion were evaluated in vitro using knockdown and ectopic expression strategies.

Results: Microarray analysis revealed that up-regulation of AFAP1-AS1 expression in PDAC tissues compared with normal adjacent tissues, which was confirmed by RT-qPCR in 69/90 cases (76.7\%). Its overexpression was associated with lymph node metastasis, perineural invasion, and poor survival. When using AFAP1-AS1 as a prognostic marker, the areas under ROC curves were 0.8669 and 0.9370 for predicting tumor progression within 6 months and 1 year, respectively. In vitro functional experiments involving knockdown of AFAP1-AS1 resulted in attenuated PDAC cell proliferation, migration, and invasion. Ectopic expression of AFAP1-AS1 promoted cell proliferation, migration, and invasion.
\end{abstract}

Conclusions: AFAP1-AS1 is a potential novel prognostic marker to predict the clinical outcome of PDAC patients after surgery and may be a rational target for therapy.

Keywords: Pancreatic ductal adenocarcinoma, AFAP1-AS1, Long noncoding RNAs

\section{Background}

Pancreatic ductal adenocarcinoma (PDAC) remains one of the most aggressive human cancers [1]. Despite substantial efforts, PDAC is associated with a short survival that has been declining steadily since the early 1990s [2]. PDAC is characterized by a highly malignant phenotype that is associated with early metastasis and resistance to chemotherapy and radiation therapy [3]. There is an urgent need for further understanding of the mechanism

\footnotetext{
* Correspondence: chent_sysu@163.com; chent_sysu@163.com

${ }^{\dagger}$ Equal contributors

'Department of Hepatobiliary Surgery, Sun Yat-sen Memorial Hospital, Sun Yat-sen University, Guangzhou, China

${ }^{2}$ Department of Pancreaticobiliary Surgery, Hepatobiliary Surgery, Sun Yat-sen Memorial Hospital, Sun Yat-sen University, Guangzhou, China

Full list of author information is available at the end of the article
}

of PDAC development and new innovative therapeutic approaches. Identifying the underlying molecular mechanisms of invasion and metastasis in PDAC will be essential for the identification of effective drug targets.

In recent years, it has become increasingly apparent that the noncoding portion of the genome is of crucial functional importance in both normal physiology and diseases [4]. Long noncoding RNAs (lncRNAs), which are defined as those longer than $\sim 200$ nucleotides but lacking protein coding capacity [3], have recently been shown to play a key role in regulating vital cellular functions including cancer progression [5]. To date, thousands of lncRNAs have been discovered through chromatin signature analysis and large-scale sequencing, and functional studies have shown that many of them

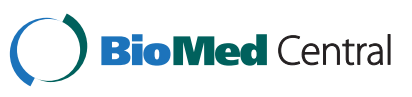


exhibit diverse biological functions and have clinical significance [6]. Importantly, many lncRNAs have been identified as being cancer-specific [5,7]. For example, aberrant expression of IncRNA HOTAIR was associated with various cancers such as breast, hepatocellular, gastric, colorectal, and pancreatic, and its expression was associated with survival and prognosis of cancer patients [8]. MALAT1 was discovered as a prognostic marker for lung cancer metastasis but also been linked to several other human malignancies [9]. Other examples include HULC in hepatocellular carcinoma [10] and PCGEM1 in prostate cancer $[11,12]$. In pancreatic, a number of lncRNAs were found to exhibit pro-oncogenic or tumor-suppressive activities, such as ENST00000480739 [13], LOC285194 [13], HULC [14], HOTAIR [15], and MALAT1 [16], suggesting an important of lncRNAs in progression of pancreatic cancer. Therefore, identification of lncRNAs involved in PDAC progression might help yield novel prognostic biomarkers or therapeutic targets.

In this study, we observed that a lncRNA, AFAP1-AS1, was substantially overexpressed in PDAC tissues. Knockdown of AFAP1-AS1 could inhibit cell proliferation, migration, and invasion of PDAC cells. Moreover, AFAP1-AS1 expression correlated with lymph node metastasis, perineural invasion, and poor survival in PDAC patients. Our results suggest that AFAP1-SA1 may represent a novel indicator of poor prognosis and a potential therapeutic target in PDAC.

\section{Methods}

\section{Cell culture}

The human pancreatic cancer cell lines Panc1 (CRL1469 ${ }^{\mathrm{mm}}$ ), MIAPaCa-2(CRL-1420 ${ }^{\mathrm{mm}}$ ), Capan2(HTB-80 ${ }^{\mathrm{mm}}$ ), SW1990(CRL-2172 $\left.2^{\mathrm{m}}\right)$, and BXPC-3 (CRL-1687 $\left.{ }^{\mathrm{mm}}\right)$, and the human pancreatic ductal epithelial cells line HPDE6 were purchased from the American Type Culture Collection and grown in complete growth medium as recommended by the supplier with $10 \%$ fetal bovine serum (FBS) and $1 \%$ penicillin/streptomycin. All cells were cultured in a humidified $5 \% \mathrm{CO}_{2}$ incubator at $37^{\circ} \mathrm{C}$.

RNA Isolation, quantitative real-time reverse-transcription polymerase chain reaction (PCR), and microarrays

Quantitative real-time PCR (RT-qPCR) was performed for AFAP1-AS1 and the epithelial-mesenchymal transition (EMT) markers E-cadherin, N-cadherin, Vimentin, Snai1, and Slug. $\beta$-Actin was used as an internal control. RNA was extracted from frozen pancreatic cancer tissues and their corresponding non-neoplastic tissues and pancreatic cell lines using TRIzol reagent (Invitrogen, Carlsbad, CA, USA). The total RNA was then converted to cDNA by reverse-transcription using oligodT primers and SuperScript II reverse transcriptase (Invitrogen). For real-time quantitative PCR, three replicates of each sample were amplified and analyzed using a Roche Light-Cycler (Roche, Basel, Switzerland). The $20 \mu \mathrm{l}$ reaction mixtures contained SYBR Green reaction mix (Qiagen Sciences) and $0.5 \mathrm{mM}$ of primer. Relative gene expression was determined using the comparative delta-delta CT method (2- $\Delta \Delta \mathrm{Ct})$. The primer sequences for PCR were provided in the supplementary materials (Additional file 1).

Transcriptomic analysis was performed using Arraystar human lncRNA microarrays, V3 (Agilent, USA), which target 27958 Entrez protein-coding genes and 7419 lncRNAs. Total RNA was extracted and mRNA was purified using the mRNA-ONLYTM Eukaryotic mRNA Isolation Kit (Epicentre). Total RNA was fragmented, labeled (One-Color, Cy3, Agilent), purified, and hybridized with probes in Hybridization Chamber gasket slides (Agilent). The slides were then washed and scanned with an Agilent Microarray Scanner. The raw data were extracted with Agilent Feature Extraction software (Agilent Technology). This software uses the robust multi-array average algorithm to adjust the background signals. Normalized data were obtained after performing the quantile method of intra-microarray normalization and the median method of baseline transformation between the microarrays. Differentially expressed genes with a raw expression level of $>400$ in more than 4 out of the 12 samples used for profiling were extracted and ordered by p-value. Genes with the highest top $10 \mathrm{p}$-values were selected for validation. The microarray platform and data were submitted to the Gene Expression Omnibus public database at the National Center for Biotechnology Information (accession number: GSE61166, http://www.ncbi.nlm.nih.gov/ geo/query/acc.cgi?acc=GSE61166).

\section{Immunoblotting}

Immunoblotting was performed as described previously [17]. Briefly, cells were washed in phosphate-buffered saline (PBS) and lysed with RIPA buffer (Invitrogen, Carlsbad, CA) plus protease inhibitor cocktail (Roche, Mannheim, Germany). For equal protein loading, a bicinchoninic acid protein assay kit (Pierce) was used to calculate protein concentration in each sample. Equivalent amounts of proteins were subjected to sodium dodecyl sulfate polyacrylamide gel electrophoresis, transferred to a polyvinylidene fluoride membrane, blocked in 5\% fat-free milk for 2 hours at room temperature, and detected with appropriate primary antibodies. The following antibodies were used for analysis: anti-E-cadherin (1:1000; BD Biosciences, CA, USA), anti-N-cadherin (1:1000; BD Biosciences), antiVimentin (1:1000; BD Biosciences), anti-slug (1:1000; Abcam), anti-snail (1:1000, Abcam), and anti- $\beta$-actin (Sigma). $\beta$-Actin was used for loading controls. 
Horseradish peroxidase-conjugated secondary antibodies (Cell Signaling Technology), and an ECL chemiluminescence kit (Pierce) were used to detect bound antibody.

\section{AFAP1-AS1 knockdown Vector construction and virus infection}

For lentivirus-mediated suppression of human AFAP1-AS1, the following shRNA and scrambled control shRNA were inserted into the pMKO.1-puro vector: \#1, forward, $5^{\prime}-\mathrm{C}$ CGGAGCGGTCTCAGCCGAATGACTCTCGAGAGTC ATTCGGCTGAGACCGCTTTTTTG-3' and reverse, 5'AATTCAAAAAAGCGGTCTCAGCCGAATGACTCTCG AGAGTCATTCGGCTGAGACCGCT-3'; \#2, forward, 5'CCGGAACACCAATCCCAAGAGGTGACTCGAGTCAC CTCTTGGGATTGGTGTTTTTTTG-3' and reverse, 5'AATTCAAAAAAACACCAATCCCAAGAGGTGACTCG AGTCACCTCTTGGGATTGGTGTT-3'; scrambled control shRNA, forward $5^{\prime}$-CCGGTTTCTCCGAACGTGTC ACGTCTCGAGACGTGACACGTTCGGAGAATTTTTG -3'and reverse, 5'-AATTCAAAAAGTTCTCCGAACGT GTCACGTCTCGAGACGTGACACGTTCGGAGAA-3'. For ectopic expression of AFAP1-AS1, The full-length AFAP1-AS1 cDNA was generated by reverse transcriptasepolymerase chain reaction (RT-PCR) using total RNA from SW1990 pancreatic cancer cell line. The AFAP1-AS1 cDNA with the wild-type sequence was inserted into the EcoRI site of the pcDNA3.1 $(+)$ expression vector (Invitrogen) to obtain the AFAP1-AS1wt/pcDNA3.1(+) construct. Lentivirus packaging, cell infection and selection of puromycinresistant cells was performed as previously described [17].

\section{Cell growth and cell cycle assays}

Cell proliferation analysis 3-(4,5-dimethylthiazol-2-yl)-2,5diphenyltetrazolium bromide (MTT) substrate (Sigma-Aldrich) was used to assay cell proliferation according to the manufacturer's instructions. Briefly, a total of $3 \times 10^{3}$ cells were seeded into 96-well dishes and allowed to adhere overnight. The growth curves of cells, covering a total of 3 days of culturing, were determined through measuring absorbance at $570 \mathrm{~nm}$. After transfection, $5 \times 10^{4} \mathrm{SW} 1990$, MIAPaCa- 2 cells, or PANC-1 cells were collected and washed three times with PBS. After RNase digestion and PI dyeing, the cells were subjected to FACS analysis.

\section{Migration and invasion assays}

The cell migration assay was performed using BD Transwell chambers. Cell invasion assays were performed with chambers uniformly covered with Matrigel (BD Biosciences) diluted with Dulbecco's modified Eagle's medium (DMEM) to a certain percentage and incubated at $37^{\circ} \mathrm{C}$ for 30 minutes. Cells $\left(1 \times 10^{6}\right)$ were suspended in $200 \mu \mathrm{l}$ serum-free DMEM medium and seeded into the upper chamber of each insert. Then, $600 \mu \mathrm{l}$ of DMEM containing
$10 \%$ FBS was added to a 24-well plate. After incubation at $37^{\circ} \mathrm{C}$ for $12 \mathrm{~h}$, the cells that migrated were fixed and stained for $20 \mathrm{~min}$ in a dye solution containing $0.4 \%$ crystal violet and $20 \%$ methanol. The cells in the upper layer of the membrane were removed and the cells in the lower layer were washed off with $33 \%$ acetic acid $(500 \mu \mathrm{l}$ per chamber). The migrated cells was quantified by measuring the absorbance of eluent at $570 \mathrm{~nm}$. The relative migration fold change of the experimental group was calculated by normalizing to that of the control group.

\section{Patient samples}

All samples were obtained from patients when undergoing resection of the pancreas at Sun Yat-Sen Memorial Hospital between 2009 and 2014. Informed consent was obtained from the patients before sample collection. All patients had a clear histological diagnosis. Patients' specimens and the related clinicopathological data, including complete follow-up, were obtained from the Institute of Pathology and from the Department of Pancreaticobiliary, Sun Yat-Sen Memorial Hospital. All patients in this study met the following criteria: 1) PDAC diagnosis was verified by pathological examination; 2) paraffinembedded tissues were well stored and qualified for serial section; 3) the corresponding tumor tissues and the paired non-tumor tissues were stored in liquid nitrogen immediately following surgical removal; 4) no anticancer treatments given before biopsy collection; and 5) availability of exhaustive clinicopathologic and follow-up data.

\section{Tumorigenicity assays in nude mice}

All experiments involving animals were conducted according to the institutional guidelines of Guangdong Province and were approved by the institutional guidelines of Guangdong Province and by the Use Committee for Animal Care. BALB/c nude mice (5 weeks old) were randomly separated into the shControl group or the shAFAP1-AS1 group (5 mice per group). SW1990 cells $\left(3 \times 10^{6}\right.$ cells/ mouse) stably transfected with shAFAP1-AS1 or control shRNA were injected subcutaneously into the right axilla of each mouse. Tumor volume was calculated using the following formula: volume $=\left(\mathrm{L} \times \mathrm{W}^{2}\right) / 2$, where $\mathrm{L}$ and $\mathrm{W}$ are the longest and shortest diameters, respectively. The mice were sacrificed two weeks after injection.

\section{Statistical analysis}

Statistical analyses were performed using SPSS Statistics 17.0 (SPSS $\left.\operatorname{lnc} c^{\circ}\right)$. The chi-square test $\left(X^{2}\right.$ test), Fisher's exact test for nonparametric variables, and Student's $t$ test for parametric variables (two-tailed) were used. Differences in patient survival were assessed using the Kaplan-Meier method and analyzed using the log-rank test in univariate analysis. All tests were two tailed, and 
results with $P=0.05$ were considered statistically significant.

\section{Results}

AFAP1-AS1 is aberrantly overexpressed in human PDAC cell lines and cancerous tissues

As a first attempt to identify differentially expressed long noncoding RNAs (lncRNAs) in two subtypes of PDAC tissues (PDAC patients with diabetes versus PDAC patients without diabetes), we conducted microarray analysis utilizing a microarray targeting 7419 LncRNAs using eight cases of PDAC tissues and four cases of chronic pancreatitis tissues (CP) (accession number: GSE61166). All of the differentially expressed ( $>$ or $<2$ fold change) lncRNAs were listed in Additional file 2. We noticed that the long noncoding RNA AFAP1-AS1 is one of the most up-regulated lncRNAs in both subtypes of PDAC tissues (Figure 1a), suggesting a potentially important role for AFAP1-AS1 in PDAC development. Therefore, we next examined the expression of AFAP1-

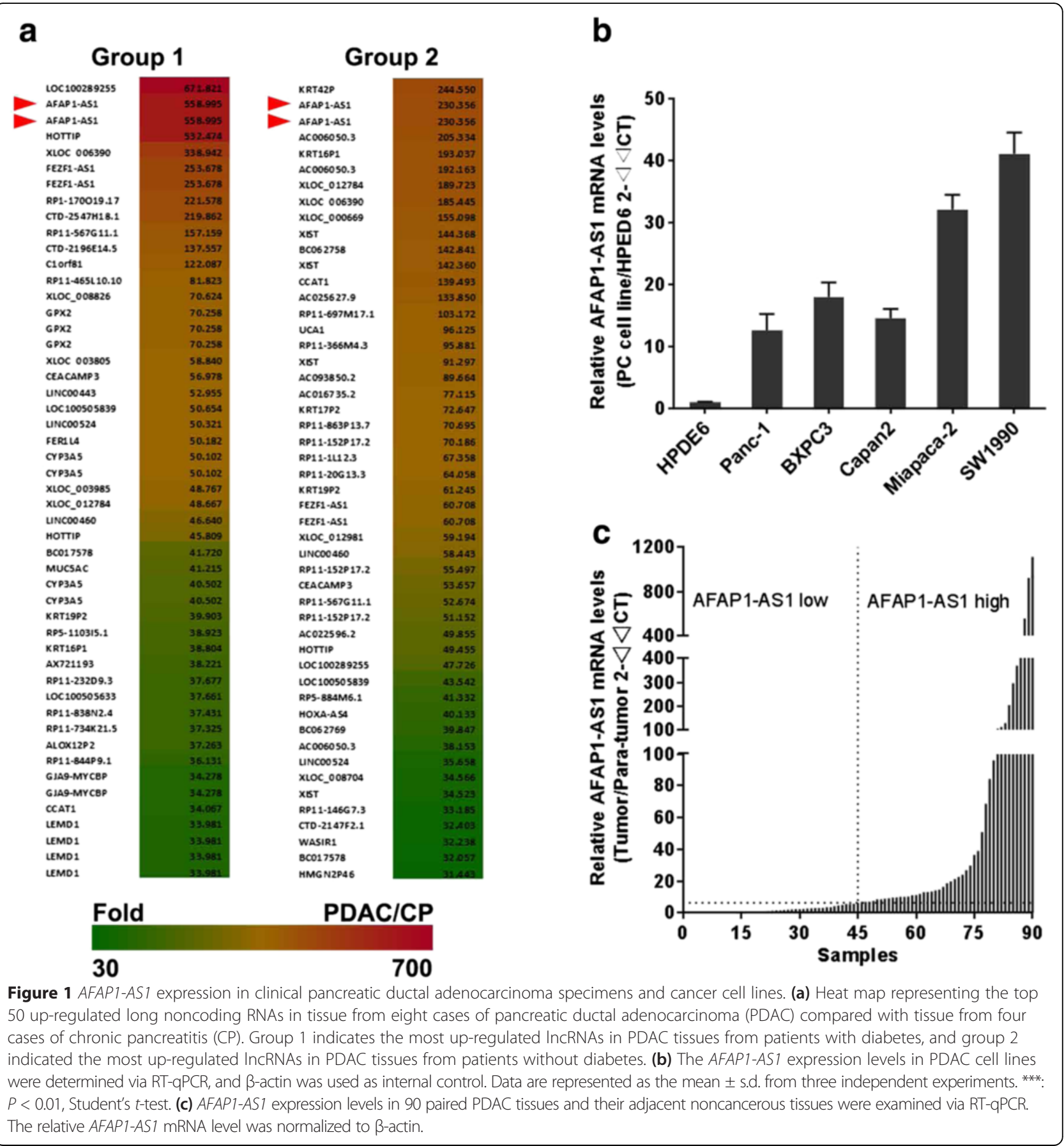



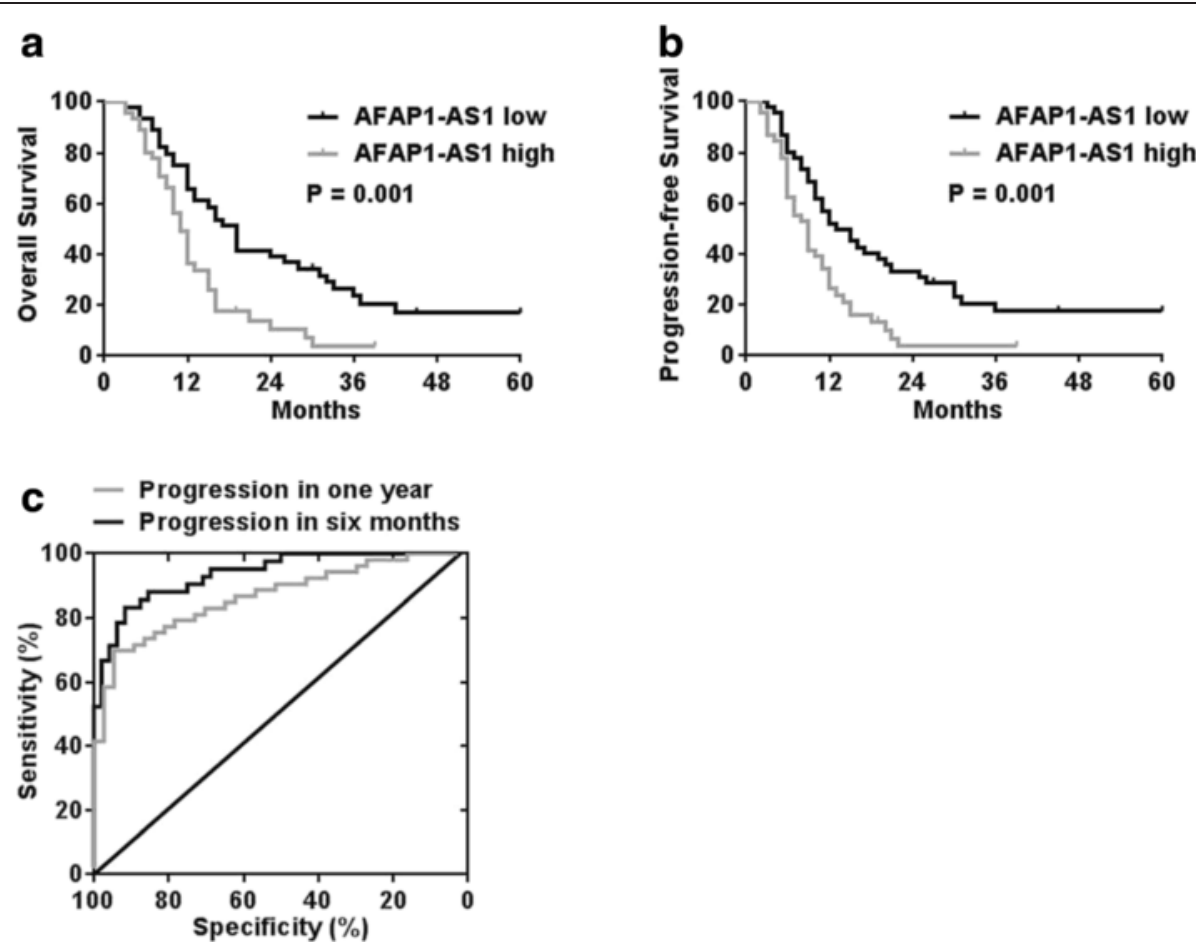

Figure 2 High AFAP1-AS1 expression predicts poor prognosis in PDAC patients with surgical resection. a) and b) Patients were equally divided into two groups based on AFAP1-AS1 mRNA levels. The log-rank test (2-sided) was used to compare differences between groups. The Kaplan-Meier curves show analyses of overall survival and progression-free survival. (c) ROC curve analysis was conducted to investigate the value of AFAP1-AS1 in predicting tumor progression (recurrence and/or metastasis) within 6 months or 1 year after surgical resection.

AS1 in multiple PDAC cell lines. We observed that the expression level of AFAP1-AS1 in each PDAC cell line was dramatically up-regulated compared with the HPDE6 cell line (human pancreatic ductal epithelium cell; Figure 1b). Finally, we sought to identify whether AFAP1-AS1 was upregulated in PDAC cell lines and in a large sample size of PDAC tissues. As shown in Figure 1c, we discovered that AFAP1-AS1 was widely upregulated in PDAC tissues compared their paired adjacent non-tumor tissues.

High AFAP1-AS1 expression predicts poor prognosis in PDAC patients with surgical resection

We next asked whether the expression of AFAP1-AS1 correlated with the clinical outcome in patients with PDAC. As shown in Figure 1c, 90 cases of PDAC patients received surgical resection were divided into two groups based on APAF1-AS1 expression with 45 patients in each group. Log-rank analysis indicated that the overall survival and progression-free survival was significantly worse in patients with higher AFAP1-AS1 expression in their tumor tissues (Figure 2a, b). Statistical analysis also revealed that AFAP1-AS1 overexpression correlated with lymph node metastasis and perineural invasion (Table 1). No statistical correlation with gender, age, tumor stage and tumor grade was observed. To further determine whether and how AFAP1-AS1 can serve as a biomarker to predict tumor
Table 1 Correlation of AFAP1-AS1 expression and clinicopathological characteristics

\begin{tabular}{|c|c|c|c|c|}
\hline \multirow[t]{2}{*}{ Factors } & & \multicolumn{2}{|c|}{ AFAP1-AS1 expression } & \multirow[t]{2}{*}{ P value* } \\
\hline & & $\begin{array}{l}\text { Higher } \\
(\mathrm{n}=45)\end{array}$ & $\begin{array}{l}\text { Lower } \\
(n=45)\end{array}$ & \\
\hline \multirow[t]{2}{*}{$\overline{\text { Age }}$} & $<60$ & 25 & 20 & 0.292 \\
\hline & $\geq 60$ & 20 & 25 & \\
\hline \multirow[t]{2}{*}{ Sex } & Male & 29 & 28 & 0.827 \\
\hline & Female & 16 & 17 & \\
\hline \multirow[t]{3}{*}{ Differentiation } & Well & 15 & 18 & 0.598 \\
\hline & Moderate & 17 & 18 & \\
\hline & Poor & 13 & 9 & \\
\hline \multirow[t]{2}{*}{ UICC stage } & $\mathrm{pl}$ & 13 & 11 & 0.634 \\
\hline & pll & 32 & 34 & \\
\hline \multirow[t]{3}{*}{ T stage } & $\mathrm{T} 1$ & 6 & 9 & 0.355 \\
\hline & $\mathrm{T} 2$ & 18 & 13 & \\
\hline & T3 & 21 & 13 & \\
\hline \multirow[t]{2}{*}{ N stage } & No & 10 & 27 & 0.001 \\
\hline & N1 & 35 & 18 & \\
\hline \multirow[t]{2}{*}{ Perineural invasion } & Negative & 18 & 29 & 0.020 \\
\hline & Positive & 27 & 16 & \\
\hline
\end{tabular}

*Pearson Chi-Square test. 
progression (local recurrence and/or metastasis) after surgery, we constructed a ROC (receiver operating characteristic) curve analysis (Figure 2c). For predicting progression within 1 year, the area under the ROC curve was 0.8669 (p $<0.0001$ ) with an optimal cutoff point of 8.797 (tumor/ para-tumor; sensitivity $=69.81 \%$, specificity $=94.59 \%)$ and for predicting progression within in 6 months, the area under the ROC curve was 0.9370 ( $\mathrm{p}<0.0001)$ with an optimal cutoff point of 8.797 (tumor/para-tumor; sensitivity $=83.33 \%$, specificity $=91.67 \%)$. These findings suggest that AFAP1-AS1 has potential diagnostic value in predicting early recurrence of PDAC.

\section{Inhibition of AFAP1-AS1 in PDAC cells leads to reduced proliferation}

To further examine whether AFAP1-AS1 has a causal role in PDAC progression, in vitro functional studies were conducted. We knocked down AFAP1-AS1 expression in MIAPaca-2 and SW1990 cells via stable transfection, and the efficiency of knockdown of the two shRNAs was evaluated (Figure 3a), the most effective shRNA \#2 was chose for the following study. AFAP1AS1 depletion resulted in decreased tumor cell proliferation both in PDAC cell line MIAPaca-2 and SW1990, as determined by MTT assay (Figure 3b, c). We also performed cell cycle assays after shRNA transfection using flow cytometry (Figure 3d, e). Results showed that suppression of AFAP1-AS1 significantly induced G2/M phase arrest. Taken together, these findings suggest that the AFAP1-AS1 modulates cell proliferation partly through regulating cell cycle.

\section{AFAP1-AS1 regulates cell migration and invasion}

Enhanced cell migration and invasion abilities are key features associated with cancer metastasis. We therefore examined whether AFAP1-AS1 knockdown affects these functions in PDAC cells. As shown in Figure 4a and b, AFAP1-AS1 knockdown significantly decreased cell motility. Similarly, a matrigel invasion assay showed that AFAP1-AS1 knockdown significantly inhibited invasiveness in MIAPaca-2 and SW1990 cells (Figure 4c, d). Since epithelial-mesenchymal transition (EMT) is closely related with the cell motility and invasiveness, we next examined whether the knockdown of AFAP1-AS1 affects the expression of EMT-related genes. Both PCR (Figure 4e and f) and Western blot analyses (Figure $4 \mathrm{~g}$ ) showed that

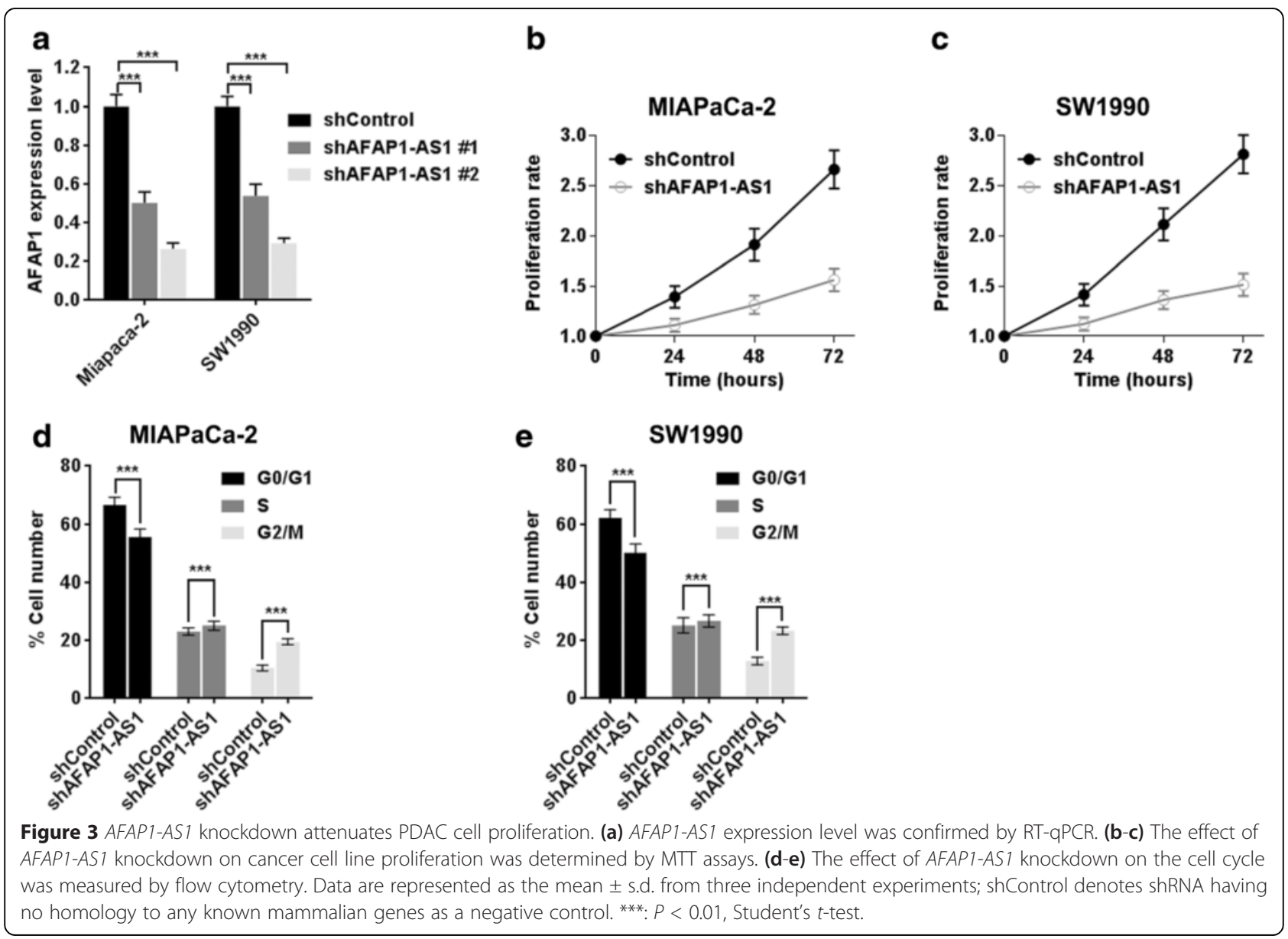




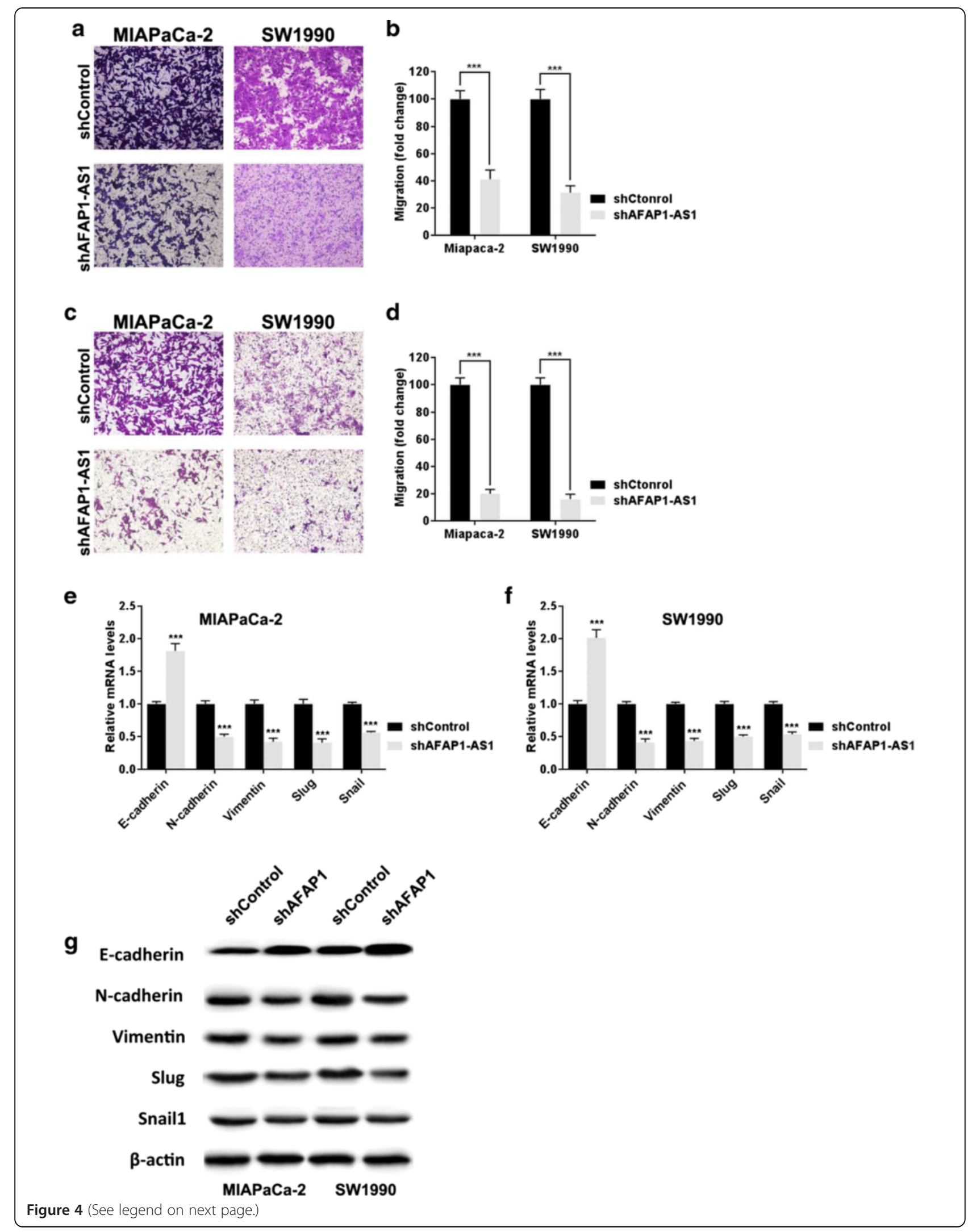


(See figure on previous page.)

Figure 4 AFAP1-AS1 knockdown inhibits migration and invasion of PDAC cells. (a) Representative images of transwell assay after AFAP1-AS1 knockdown in PDAC cell line MIAPaCa-2 and SW1990. (b) Analysis of OD (570 nm) absorbance of crystal violet-stained cells in transwell assay. (c) Representative images of Matrigel invasion assay after AFAP1-AS1 knockdown in PDAC cell line MIAPaCa-2 and SW1990. (d) Analysis of OD (570 $\mathrm{nm}$ ) absorbance of crystal violet-stained cells in Matrigel invasion assay. (e-f) Relative mRNA expression levels of EMT-related genes (normalized to B-actin) in PDAC cells after AFAP1-AS1 knockdown were determined by RT-qPCR. (g) Western blot analysis of E-cadherin, N-cadherin, and Vimentin. Data are represented as the mean \pm s.d. from three independent experiments, shControl denotes shRNA having no homology to any known mammalian genes as a negative control. ${ }^{* * *}: P<0.01$, Student's t-test.

suppression of AFAP1-AS1 in PDAC cells was associated with upregulation of epithelial marker E-cadherin and downregulation of mesenchymal markers.

\section{Ectopic expression of AFAP1-AS1 promoted proliferation, migration, and invasion of pancreatic cancer cell}

To further evaluate the oncogenic role of AFAP1-AS1 in PDAC, ectopic expression of AFAP1-AS1 was carried out in PANC-1 cells which have the lowest AFAP1-AS1 level among the five pancreatic cancer cells. Overexpression of AFAP1-AS1 was confirmed by qRT-PCR (Figure 5a). As expected, AFAP1-AS1 overexpression resulted in increased tumor cell proliferation (Figure $5 \mathrm{~b}$ ). In addition, migration assay and invasion assay were also performed (Figure 5c), and PANC-1 cells showed significant enhanced migration and invasion ability after AFAP1-AS1 overexpression (Figure 5d). Both PCR (Figure 5e) and Western blot analyses (Figure 5f) demonstrated that introduction of AFAP1-AS1 in PANC-1 cells was associated with down of epithelial marker E-cadherin and upregulation of mesenchymal markers.

\section{Inhibition of AFAP1-AS1 impaired pancreatic cancer cell tumorigenicity in vivo}

To evaluate the effect of AFAP1-AS1 on the efficiency of xenograft formation of pancreatic cancer cells, we analyzed the in vivo tumorigenicity of SW1990 cells in nude mice following the shRNA-mediated knockdown of AFAP1-AS1. As expected, both tumor volume (Figure 6a, b) and tumor weight (Figure 6c) were significant decreased with pancreatic cancer cells when AFAP1-AS1 expression was inhibited.

\section{Discussion}

Although thousands of lncRNAs have recently been identified, investigation of their respective roles in modulating gene expression is relatively incomplete. Functional studies have indicated that some lncRNAs are involved in human cancer tumorigenesis, progression, and adjuvant therapy resistance, acting as oncogenes or tumor suppressors $[4,18]$. In the current study, by using high-throughput microarrays, we found that AFAP1-AS1 is markedly upregulated in PDAC cell lines and in primary material, and its overexpression correlates with lymph node metastasis, perineural invasion, and poor prognosis of PDAC patients. These observations suggest pro-oncogenic activity of $A F A P 1-A S 1$, a notion that is further supported by our functional studies showing that AFAP1-AS1 knockdown attenuates PDAC cell proliferation, migration, and invasion.

Lymph node metastasis and perineural invasion are the strongest indicators of short overall survival in PDAC patients. Efforts have recently been made to identify molecular predictive factors in pancreatic cancer patients [19-21]. In the current study, we observed that AFAP1-AS1 overexpression is associated with lymph node metastasis, perineural invasion, and overall survival after surgical treatment, raising the possibility that this lncRNA may provide a means of identifying high-risk patients for more intensive therapy. Importantly, ROC curve analysis revealed that AFAP1-AS1 has great potential in predicting tumor progression after surgery. In the data set of our present study, we observed that an over 8-fold increase in AFAP1-AS1 expression in PDAC tissues compared with adjacent non-tumor tissues was associated with an extremely high risk of short-term recurrence. Whether AFAP1-AS1 alone, or in combination with other markers, could predict PDAC shortterm recurrence required further research through well-designed studies with larger sample size.

In this study, the clinical value of AFAP1-AS1 in PDAC was supported by functional analysis, which showed that AFAP1-AS1 suppression diminished migration, invasion, and expression of EMT-related genes in PDAC cells, and AFAP1-AS1 ectopic expression promoted these malignant behaviors vice versa. This long noncoding RNA was first reported by $\mathrm{Wu}$ et al. [22], who showed that AFAP1-AS1 is overexpressed in primary esophageal adenocarcinoma tissues and regulates esophageal adenocarcinoma cell proliferation, migration, and invasion. Consistent with the latter report, the findings of our study support a similar oncogenic role for AFAP1-AS1 in PDAC. Importantly, our data showed that AFAP1-AS1 was one of the most intensely and frequently overexpressed lncRNA in PDAC, further highlighting this transcript to be of significant biological interest in the study of pancreatic cancer pathogenesis.

The AFAP1-AS1 is derived from the antisense strand of the AFAP1 (Actin Filament Associated Protein) gene, the sense strand of which encodes the protein AFAP1. 


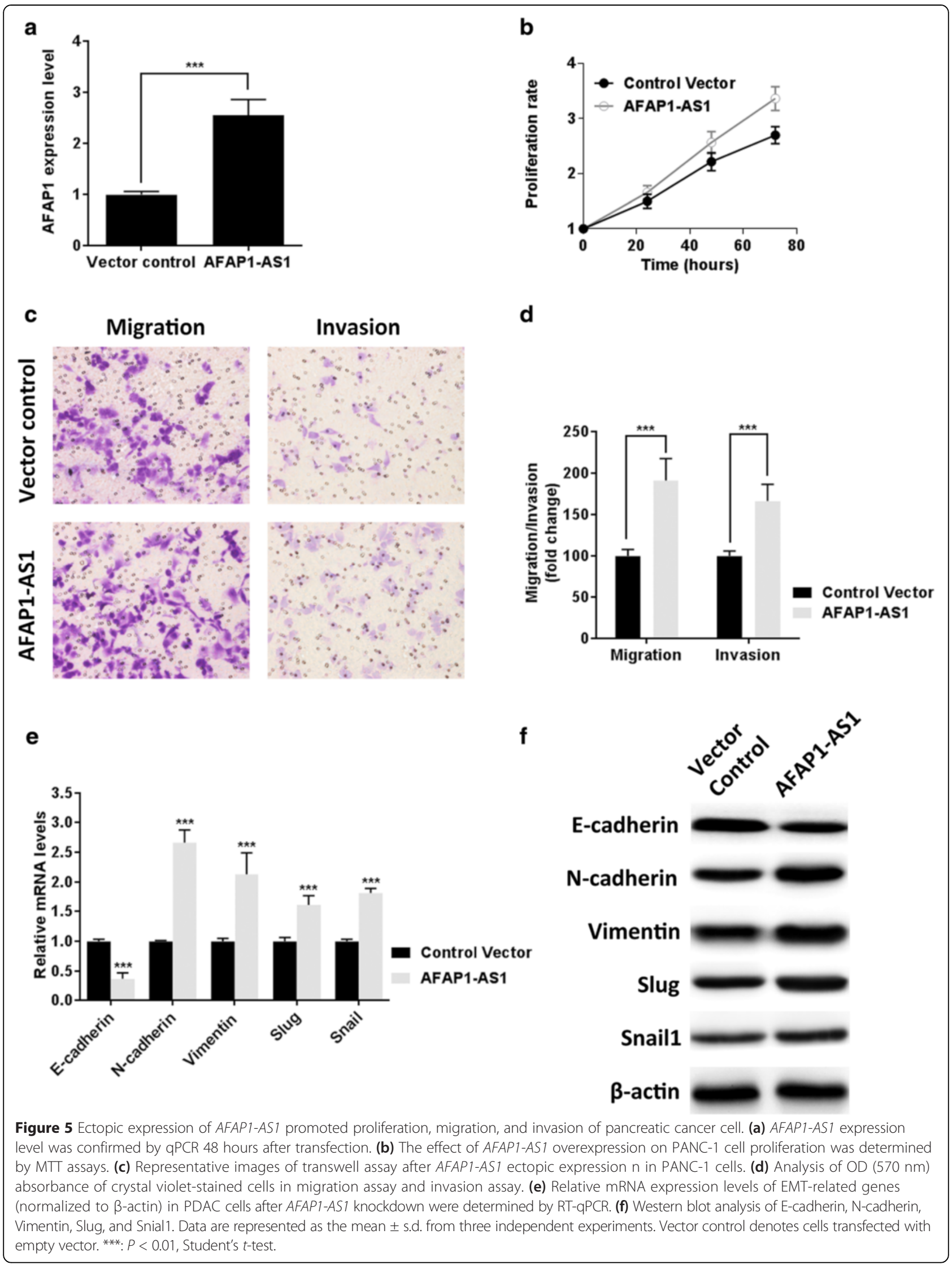



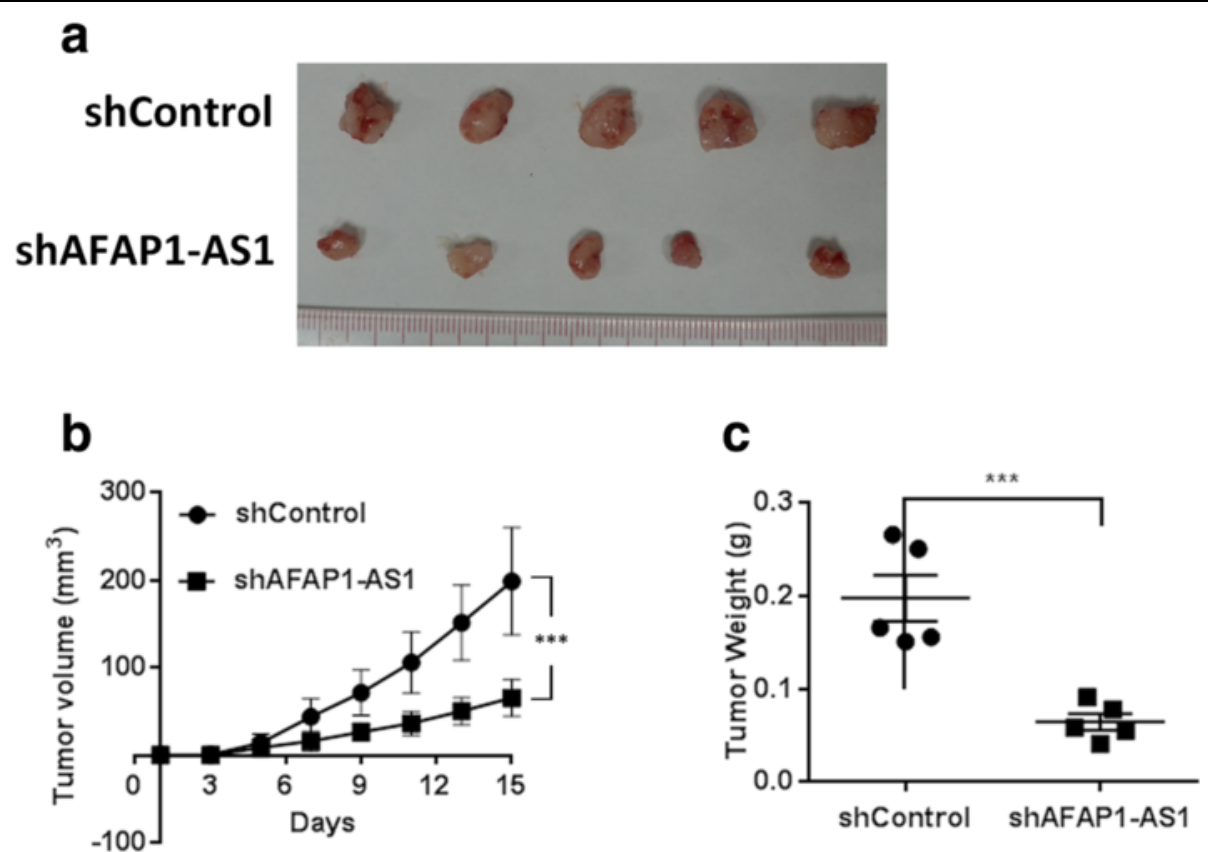

Figure 6 Inhibition of AFAP1-AS1 impaired pancreatic cancer cell tumorigenicity in vivo. (a) (b) Nude mice were subcutaneously injected into the right axilla with $3 \times 10^{6}$ cells infected with shControl lentiviral vector (containing scrambled control shRNA) or shAFAP1-AS1 lentiviral vector (containing shRNA targeting AFAP1-AS1). Tumor growth was then monitored using calipers, and the mice were killed two weeks after injection. (c) The tumors were weighed, and compared between the groups. ***: $P<0.01$, Student's $t$-test.

The function of the AFAP1 gene in oncogenesis has been investigated in both breast and prostate cancer. It has been reported that AFAP1 regulates Src activity and promotes the formation of actin stress fibers and focal adhesions in breast cancer cells [23]. The loss of AFAP1 in prostate cancer cells reduced cell proliferation and tumorigenesis in nude mice [24]. Usually, the antisense RNA regulates expression of its cognate sense gene, but Wu et al. demonstrated that AFAP1-AS1 had limited effect on AFAP1 expression, instead functioning in an almost AFAP1-independent manner [22]. Therefore, it will be of great interest in future to investigate whether AFAP1-AS1 is involved in regulating AFAP1. Whether overexpression of AFAP1-AS1 is induced by hypomethylation or other epigenetic mechanism also deserves further study.

\section{Conclusions}

In summary, we have identified that a long noncoding RNA, AFAP1-AS1, is up-regulated in PDAC tissues and serves as a negative prognostic factor for lymph node metastasis, perineural invasion, and poor survival in PDAC patients. The value of AFAP1-AS1 as a potential prognostic biomarker and/or therapeutic target in PDAC was supported by findings that knockdown of AFAP1AS1 in PDAC cells inhibits cell proliferation, motility, and invasiveness in vitro.

\section{Additional files}

Additional file 1: The primers used for the qRT-PCR analysis.

Additional file 2: Differentially expressed IncRNAs between PDAC tissues and CP tissues.

\section{Abbreviations}

PC: Pancreatic cancer; PDAC: Pancreatic ductal adenocarcinoma; CP: Chronic pancreatitis; IncRNA: Long noncoding RNA; AFAP1: Actin filament associated protein 1; AFAP1-AS1: Actin filament associated protein 1 antisense RNA; EMT: Epithelial Mesenchymal Transition.

\section{Competing interests}

The authors declare that they have no competing interests.

\section{Authors' contributions}

RF Chen and Tao Chen conceived and carried out the experiments, analyzed and interpreted the data and drafted the manuscript. Yibiao Ye and Jie Chen conceived the experiments, analyzed and interpreted data and wrote the manuscript. Y Zhou, ZQ Fu, QB Zhou, YX Wang, WC Gao, SY Zheng, and XH Zhao participated in the experiment and helped analyzed data. All authors read and approved the final manuscript.

\section{Acknowledgments}

This work was supported by the National Natural Scientific Foundation of China (No. 81000917 and No. 81370059). The funders had no role in the study design, data collection and analysis, decision to publish, or preparation of the manuscript. We thank Prof. Gu Jing (School of Public Health, Sun Yatsen University) for providing suggestions of statistics.

We demonstrate for the first time that a long noncoding RNA, AFAP1-AS1, is extremely elevated in PDAC tissues. We show that increased expression of AFAP1-AS1 in PDAC tissues predicts very poor survival and a shorter time to tumor progression. In vitro functional analysis supported an oncogenic role for this InCRNA in PDAC, providing a rational basis for its predictive value. 


\section{Author details}

'Department of Hepatobiliary Surgery, Sun Yat-sen Memorial Hospital, Sun Yat-sen University, Guangzhou, China. '2Department of Pancreaticobiliary Surgery, Hepatobiliary Surgery, Sun Yat-sen Memorial Hospital, Sun Yat-sen University, Guangzhou, China. ${ }^{3}$ Department of Medical Oncology, Sun Yat-sen Memorial Hospital, Sun Yat-sen University, Guangzhou, China. ${ }^{4}$ Department of General Surgery, The Second Affiliated Hospital of Sun Yat-sen University, Sun Yat-sen University, 107 Yan-Jiang Xi Road, Guangzhou 510120, China. ${ }^{5}$ Present Address: Department of General Surgery,

Guangdong General Hospital, Guangzhou, China.

\section{Received: 17 December 2014 Accepted: 14 April 2015}

\section{Published online: 30 April 2015}

\section{References}

1. Siegel R, Naishadham D, Jemal A. Cancer statistics, 2013. CA Cancer J Clin. 2013:63:11-30.

2. Ma J, Jemal A. The rise and fall of cancer mortality in the USA: why does pancreatic cancer not follow the trend? Future Oncol. 2013;9:917-9.

3. Ponting CP, Oliver PL, Reik W. Evolution and functions of long noncoding RNAs. Cell. 2009;136:629-41.

4. Mercer TR, Dinger ME, Mattick JS. Long non-coding RNAs: insights into functions. Nat Rev Genet. 2009;10:155-9.

5. Seton-Rogers S. Non-coding RNAs: the cancer $X$ factor. Nat Rev Cancer. 2013;13:224-5.

6. Hung T, Chang HY. Long noncoding RNA in genome regulation: prospects and mechanisms. RNA Biol. 2010;7:582-5.

7. Zhang H, Chen Z, Wang X, Huang Z, He Z, Chen Y. Long non-coding RNA: a new player in cancer. J Hematol Oncol. 2013;6:37.

8. Cai B, Song XQ, Cai JP, Zhang S. HOTAIR: a cancer-related long non-coding RNA. Neoplasma. 2014;61:379-91.

9. Gutschner T, Hammerle M, Diederichs S. MALAT1 - a paradigm for long noncoding RNA function in cancer. J Mol Med (Berl). 2013;91:791-801.

10. Du Y, Kong G, You X, Zhang S, Zhang T, Gao Y, et al. Elevation of highly up-regulated in liver cancer (HULC) by hepatitis $B$ virus $X$ protein promotes hepatoma cell proliferation via down-regulating p18. J Biol Chem. 2012:287:26302-11.

11. Srikantan V, Zou Z, Petrovics G, Xu L, Augustus M, Davis L, et al. PCGEM1, a prostate-specific gene, is overexpressed in prostate cancer. Proc Natl Acad Sci U S A. 2000;97:12216-21.

12. Xue B, He L. An expanding universe of the non-coding genome in cancer biology. Carcinogenesis. 2014;35:1209-16.

13. Ding YC, Yu W, Ma C, Wang Q, Huang CS, Huang T. Expression of long non-coding RNA LOC285194 and its prognostic significance in human pancreatic ductal adenocarcinoma. Int J Clin Exp Pathol. 2014;7:8065-70.

14. Peng W, Gao W, Feng J. Long noncoding RNA HULC is a novel biomarker of poor prognosis in patients with pancreatic cancer. Med Oncol. 2014:31:346.

15. Kim K, Jutooru I, Chadalapaka G, Johnson G, Frank J, Burghardt R, et al. HOTAIR is a negative prognostic factor and exhibits pro-oncogenic activity in pancreatic cancer. Oncogene. 2013;32:1616-25.

16. Jiao F, Hu H, Han T, Yuan C, Wang L, Jin Z, et al. Long noncoding RNA MALAT-1 enhances stem cell-like phenotypes in pancreatic cancer cells. Int J Mol Sci. 2015;16:6677-93

17. Tan L, Ye X, Zhou Y, Yu M, Fu Z, Chen R, et al. Macrophage migration inhibitory factor is overexpressed in pancreatic cancer tissues and impairs insulin secretion function of beta-cell. J Transl Med. 2014;12:92.

18. Gutschner T, Diederichs S. The hallmarks of cancer: a long non-coding RNA point of view. RNA Biol. 2012:9:703-19.

19. Sun $T$, Kong $X, D u Y, L i Z$. Aberrant MicroRNAs in pancreatic cancer: researches and clinical implications. Gastroenterol Res Pract. 2014:2014:386561.

20. Xiao Z, Luo G, Liu C, Wu C, Liu L, Liu Z, et al. Molecular mechanism underlying lymphatic metastasis in pancreatic cancer. Biomed Res Int 2014;2014:925845.

21. Bapat AA, Hostetter G, Von Hoff DD, Han H. Perineural invasion and associated pain in pancreatic cancer. Nat Rev Cancer. 2011;11:695-707.

22. Wu W, Bhagat TD, Yang $X$, Song JH, Cheng $Y$, Agarwal $R$, et al. Hypomethylation of noncoding DNA regions and overexpression of the long noncoding RNA, AFAP1-AS1, in Barrett's esophagus and esophageal adenocarcinoma. Gastroenterology. 2013;144:956-66.
23. Dorfleutner A, Stehlik C, Zhang J, Gallick GE, Flynn DC. AFAP-110 is required for actin stress fiber formation and cell adhesion in MDA-MB-231 breast cancer cells. J Cell Physiol. 2007;213:740-9.

24. Zhang J, Park SI, Artime MC, Summy JM, Shah AN, Bomser JA, et al. AFAP-110 is overexpressed in prostate cancer and contributes to tumorigenic growth by regulating focal contacts. J Clin Invest. 2007;117:2962-73.

\section{Submit your next manuscript to BioMed Central and take full advantage of:}

- Convenient online submission

- Thorough peer review

- No space constraints or color figure charges

- Immediate publication on acceptance

- Inclusion in PubMed, CAS, Scopus and Google Scholar

- Research which is freely available for redistribution

Submit your manuscript at www.biomedcentral.com/submit
C Biomed Central 